Europhysics Letters

PREPRINT

\title{
Pseudo-Casimir force in confined nematic polymers
}

\author{
J. Dobnikar ${ }^{2}$ and R. Podgornik ${ }^{1,2}\left(^{*}\right)$ \\ 1 Department of Physics, Faculty of Mathemathics and Physics, University of Ljubljana, \\ Jadranska 19, 1000 Ljubljana, Slovenia \\ 2 J. Stefan Institute, University of Ljubljana, Jamova 39, 1000 Ljubljana, Slovenia \\ PACS. 30.-v - Liquid crystals. \\ PACS. 41.+e - Polymers, elastomers and plastics.
}

\begin{abstract}
We investigate the pseudo-Casimir force in a slab of material composed of nematically ordered long polymers. We write the total mesoscopic energy together with the constraint connecting the local density and director fluctuations and evaluate the corresponding fluctuation free energy by standard methods. It leads to a pseudo-Casimir force of a different type than in the case of standard, short molecule nematic. We investigate its separation dependence and its magnitude and explicitly derive the relevant limiting cases.
\end{abstract}

Casimir effect is due to constrained fluctuations in media with long ranged correlations [1]. The physical nature of the medium is not particularly important. Though the standard Casimir effect has been introduced for constrained electromagnetic field fluctuations [2] it has been realized that other systems with long-range correlations exhibit a similar type of fluctuation driven interactions. Most notably critical fluids [3], smectic manifolds [4] and liquid crystals [5, all of them being prime examples of correlated fluids, give rise to a pseudoCasimir effect which comes about through constrained thermal (as opposed to quantum) fluctuations of order parameters. The nature of these order parameters of course depends on the system under study but they all exhibit massless fluctuation spectra that eventually lead to long-ranged fluctuation interactions.

Delimiting ourselves to the case of liquid crystals, the pseudo-Casimir effect has been researched in nematic, smectic and columnar uniformly ordered systems []]. In the case of inhomogeneous or frustrated order as in the case of the hybrid-aligned cell characterized by opposing surface fields or in the Fréedericksz cell where frustration arises from competing bulk and surface fields [6], the interaction induced by director fluctuations is enhanced substantially by frustration, the enhancement being progressively stronger as the system approaches the transition from uniform to distorted structure.

There seems to be some experimental evidence that backs up these theoretical predictions [7, 81 but there still lacks a definitive proof of the observability of the pseudo-Casimir interaction. It appears however that in the case of spinodal dewetting of $5 \mathrm{CB}$ on a silicon wafer [9] the pseudo-Casimir interaction is essential in giving a consistent interpretation of experimental

$\left(^{*}\right)$ To whom correspondence should be addressed. rudolf.podgornik@fiz.uni-lj.si

(c) EDP Sciences 
data, implying that this experiment can be regarded as the first observation of the pseudoCasimir effect in liquid crystals [10].

In this contribution we shall investigate the pseudo-Casimir effect in the realm of confined polymers. As a first case study we will take a nematic polymeric liquid crystal such as one can observe in stiff polyelectrolytes above the isotropic - nematic transition, confined between two apposed planar surfaces. A physical realization of this case would be a nematically ordered DNA confined between two surfaces or simply cut to form a slab of macroscopically oriented sample [11. We believe that this case is particularly instructive since we will be able to connect different limiting results with the existing calculations for ordinary, i.e. short chain, nematics [5]. It will be shown that the polymeric nature of the nematogens gives rise to additional features of the pseudo-Casimir effect that distinguish it qualitatively from results derived in the case of short nematogens. Also we will show that in the case of nematic polymers the pseudo-Casimir interaction depends on the equation of state [11] of the polymer nematic through its compressibility.

The elastic deformation of an ordinary short molecule nematic 12] with long range orientational order along the axis $\mathrm{z}$, can be described with an average director $\mathbf{n}(\mathbf{r})$, with small fluctuations in the $(x, y)$ directions: $\mathbf{n}(\mathbf{r}) \simeq\left(1, \delta n_{x}, \delta n_{y}\right)$. In this case, if the splay, twist and bend elastic constants are the Frank constants $K_{1}, K_{2}$ and $K_{3}$ one obtains for the mesoscopic elastic hamiltonian

$$
\mathcal{H}_{\mathbf{n}}=\frac{1}{2} \int d^{2} \mathbf{r}_{\perp} d z\left[K_{1}\left(\boldsymbol{\nabla}_{\perp} \cdot \delta \mathbf{n}\right)^{2}+K_{2}\left(\boldsymbol{\nabla}_{\perp} \times \delta \mathbf{n}\right)^{2}+K_{3}\left(\partial_{z} \delta \mathbf{n}\right)^{2}\right] .
$$

Besides fluctuations in nematic director one should also consider the fluctuations in local density of the molecules. For short nematogens the director and density fluctuations are decoupled and one need not consider this part of the mesoscopic free energy explicitly. For long, polymer nematics the situation is altogether different. In this case we have to consider the part of the free energy due to nonhomogeneous density fluctuations $\rho(\mathbf{r}) \simeq \rho_{0}+\delta \rho(\mathbf{r})$ in the Ornstein-Zernicke form

$$
\mathcal{H}_{\rho}=\frac{1}{2} \int d^{2} \mathbf{r}_{\perp} d z\left[B(\delta \rho)^{2}+B \xi^{2}(\nabla \delta \rho)^{2}\right],
$$

where $B$ is the osmotic compressibility modulus and $\xi$ is the density correlation length. Because polymers are here considered to be infinitely long one also has to consider the fact that director inhomogeneities can relax only if accompanied by the simultaneous density relaxation 13. This leads to the constraint

$$
0=\nabla(\rho \mathbf{n}) \simeq \partial_{z} \delta \rho+\rho_{0}\left(\nabla_{\perp} \cdot \delta \mathbf{n}\right) .
$$

Adding all the components of the mesoscopic free energy and taking the constraint Eq. 3 into account via a Lagrange multiplier we obtain

$$
\begin{aligned}
\mathcal{H}=\frac{1}{2} \int d^{2} \mathbf{r}_{\perp} d z \quad & {\left[K_{1}\left(\boldsymbol{\nabla}_{\perp} \cdot \delta \mathbf{n}\right)^{2}+K_{2}\left(\boldsymbol{\nabla}_{\perp} \times \delta \mathbf{n}\right)^{2}+K_{3}\left(\partial_{z} \delta \mathbf{n}\right)^{2}+\right.} \\
& \left.+B(\delta \rho)^{2}+B \xi^{2}(\boldsymbol{\nabla} \delta \rho)^{2}+C\left(\partial_{z} \delta \rho+\rho_{0}\left(\boldsymbol{\nabla}_{\perp} \cdot \delta \mathbf{n}\right)\right)^{2}\right] .
\end{aligned}
$$

Writing $\delta \mathbf{n}$ in the Helmholtz ansatz $\delta \mathbf{n}=\delta \mathbf{n}_{\|}+\delta \mathbf{n}_{\perp}$ we realize that the tangential part of the hamiltonian is decoupled from the constraint and its contribution to the fluctuational free energy should be the same as for short nematics [5.

Obviously in the case of polymer nematics the longitudinal director and the density field fluctuations are coupled. The fluctuational free energy is now obtained via

$$
\mathcal{F}=-k T \ln \mathcal{Z}=-k T \ln \int_{\delta \mathbf{n}(\partial)=0} \mathcal{D} \delta \mathbf{n} \int_{\delta \rho(\partial)=0} \mathcal{D} \delta \rho \exp (-\beta \mathcal{H}),
$$


where we have assumed that at the boundary of the sample the fluctuations in density and director field are quenched (i.e. $\delta \mathbf{n}(\partial)=0, \delta \rho(\partial)=0$ ). In principle one could also introduce the anchoring and tension energy of the bounding surfaces which would not bring any new qualitative features into our discussion but would make the computations less transparent.

We now analyze the fluctuational free energy of a slab of nematic material bounded at $z= \pm L / 2$ where the average director is aligned with the $z$ axis. In the case of short nematogens $(C=0)$ the free energy decouples and we obtain two contributions. A long range pseudoCasimir free energy due to director fluctuations and a short range term due to screened density fluctuations. Thus

$$
\mathcal{F}(C=0)=-\frac{k T S}{16 \pi}\left(\frac{K_{3}}{K_{1}}+\frac{K_{2}}{K_{1}}\right) \frac{\zeta(3)}{L^{2}}-\frac{k T}{16 \pi} \frac{\epsilon(L / \xi)}{L^{2}},
$$

where $S$ is the area of the surface, $\zeta(n)$ the Riemann's Zeta function and

$$
\epsilon(L / \xi)=\int_{2 L / \xi}^{\infty} u d u \ln \left(1-e^{-u}\right)
$$

Since $L / \xi \gg 1$ and the function $\epsilon(x)$ has an exponential behavior for large values of the argument the density fluctuations make a negligible contribution to the total free energy. Our result thus evidently reduces to the one obtained by Ajdari et al. [5].

In the case that the director and the density fluctuations are coupled the calculation of the functional integral Eq. 5 becomes more complicated. First of all we transform the hamiltonian Eq. A by introducing

$$
\delta \rho=\rho_{0} \nabla_{\perp} \cdot \mathbf{a}+f \quad, \quad \delta \mathbf{n}=-\partial_{z} \mathbf{a}=-\dot{\mathbf{a}} .
$$

The coupling term in this case reduces to

$$
C\left(\partial_{z} \rho+\rho_{0} \nabla_{\perp} \cdot \delta \mathbf{n}\right)^{2} \rightarrow C \rho_{0}^{2}\left(\partial_{z} f\right)^{2}=C^{\prime} \dot{f}^{2}
$$

and the hamiltonian is

$$
\begin{aligned}
& \mathcal{H}=\frac{1}{2} \int d^{2} \mathbf{r}_{\perp} d z\left[K_{3} \ddot{\mathbf{a}}^{2}+K_{1}\left(\nabla_{\perp} \dot{\mathbf{a}}\right)^{2}+B\left(\nabla_{\perp} \mathbf{a}+f\right)^{2}+B \xi^{2}\left(\nabla_{\perp}\left(\nabla_{\perp} \mathbf{a}+f\right)\right)^{2}+\right. \\
& \left.+B \xi^{2}\left(\nabla_{\perp} \dot{\mathbf{a}}+\dot{f}\right)^{2}+C^{\prime} \dot{f}^{2}\right]
\end{aligned}
$$

Clearly in the limit of strictly enforced density-director coupling $C \longrightarrow \infty$ and as a consequence $f$ becomes independent of $z$, thus $\dot{f}=0$. In this case we can introduce the following linear transformation

$$
\mathbf{a} \longrightarrow \mathbf{a}+\nabla_{\perp} A, \quad \nabla_{\perp}^{2} A=-f,
$$

obtaining finally for the hamiltonian in the limit of strong coupling

$$
\mathcal{H}=\frac{1}{2} \int d^{2} \mathbf{r}_{\perp} d z\left[K_{3} \ddot{\mathbf{a}}^{2}+K_{1}\left(\boldsymbol{\nabla}_{\perp} \cdot \dot{\mathbf{a}}\right)^{2}+B\left(\boldsymbol{\nabla}_{\perp} \cdot \mathbf{a}\right)^{2}+B \xi^{2}\left(\boldsymbol{\nabla}_{\perp}\left(\boldsymbol{\nabla}_{\perp} \cdot \mathbf{a}\right)\right)^{2}+B \xi^{2}\left(\boldsymbol{\nabla}_{\perp} \cdot \dot{\mathbf{a}}\right)^{2}\right] .
$$

Since $\mathbf{a}$ is a longitudinal vector (Eq. 8) one obtains in the Fourier space 


$$
\mathcal{H}=\frac{1}{2} \sum_{\mathbf{Q}} \int d z\left[K_{3} \ddot{\mathbf{a}}_{\|}^{2}+\left(K_{1}+B \xi^{2}\right) Q^{2} \dot{\mathbf{a}}_{\|}^{2}+B\left(1+\xi^{2} Q^{2}\right) Q^{2} \mathbf{a}_{\|}^{2}\right]
$$

The mesoscopic hamiltonian obviously corresponds to a persistent oscillator, i.e. an oscillator with an additional fourth order term in the derivatives. Since the different Fourier components are decoupled the evaluation of the functional integral proceeds straightforward and can in fact be reduced to a Feynman integral for second-derivative Lagrangian solved exactly by Kleinert 14, 15]. Taking into account the boundary conditions for the fluctuating fields we obtain up to a multiplicative constant

$$
\mathcal{Z}=\frac{(2 \pi k T)^{-1} \sqrt{\omega_{1} \omega_{2}}\left|\omega_{1}^{2}-\omega_{2}^{2}\right|\left(\omega_{1}^{2}+\omega_{2}^{2}\right)^{-1}}{\sinh \left(\omega_{1} K_{3}^{-1 / 3} L\right) \sinh \left(\omega_{2} K_{3}^{-1 / 3} L\right)-\frac{2 \omega_{1} \omega_{2}}{\left(\omega_{1}^{2}+\omega_{2}^{2}\right)}\left(\cosh \left(\omega_{1} K_{3}^{-1 / 3} L\right) \cosh \left(\omega_{2} K_{3}^{-1 / 3} L\right)-1\right)}
$$

where

$$
\begin{aligned}
\left(\omega_{1}^{2}+\omega_{2}^{2}\right) & =\frac{1}{2}\left(K_{1}+B \xi^{2}\right) Q^{2} K_{3}^{-1 / 3} \\
\left(\omega_{1} \omega_{2}\right)^{2} & =B\left(1+\xi^{2} Q^{2}\right) Q^{2} K_{3}^{1 / 3} .
\end{aligned}
$$

Introducing now $\Omega_{ \pm}=K_{3}^{-1 / 3}\left(\omega_{1} \pm \omega_{2}\right)$ and with

$$
\Omega_{ \pm}^{2}(Q)=\frac{K_{1}+B \xi^{2}}{2 K_{3}} Q\left[Q \pm 4 \Lambda \sqrt{1+\xi^{2} Q^{2}}\right] .
$$

where

$$
\Lambda=\frac{\sqrt{B K_{3}}}{K_{1}+B \xi^{2}}
$$

we obtain for the regularized fluctuation free energy per unit area, where the formally divergent bulk and surface free energy terms have already been subtracted

$$
\mathcal{F}=\frac{k T S}{4 \pi} \int_{0}^{\infty} Q d Q \ln \left(1-2 \frac{\Omega_{+}^{2}}{\Omega_{-}^{2}} e^{-\Omega_{+} L}\left[\cosh \left(\Omega_{-} L\right)-\frac{\Omega_{+}^{2}-\Omega_{-}^{2}}{\Omega_{+}^{2}}\right]+e^{-2 \Omega_{+} L}\right) .
$$

In the above equations $\Omega_{-}^{2}$ can become negative for sensible values of the correlation length $\xi$. In this case the $\cosh \Omega_{-} L$ has to be replaced by $\cos \left|\Omega_{-}\right| L$ but the integral contains no dangerous divergencies and can be evaluated straightforwardly. By introducing

$$
\mathcal{F}^{*}=\frac{k T S}{4 \pi} \Lambda^{2} \quad \text { and } \quad L^{*}=\sqrt{\frac{2\left(K_{1}+B \xi^{2}\right)}{B}}
$$

we realize that the dimensionless free energy $\mathcal{F} / \mathcal{F}^{*}$ obtained from Eq. 18 depends only on dimensionless separation $L / L^{*}$ and the dimensionless coupling constant $\gamma=\Lambda \xi$. This coupling constant basically represents the ratio between the density correlation length $\xi$ and the polymer nematic correlation length perpendicular to the average director $\Lambda^{-1}$ [11]. 


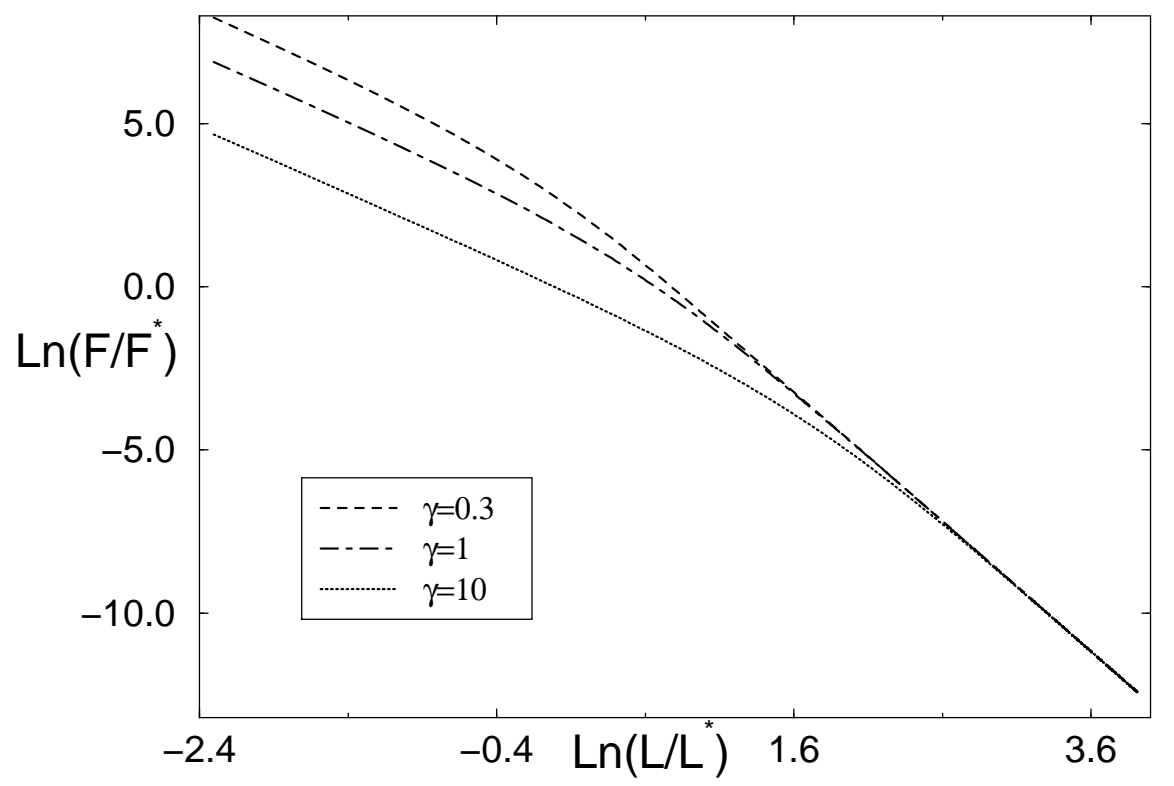

Fig. 1 - The dependence of the dimensionless free energy $\mathcal{F}^{*}$ on the dimensionless separation $\frac{L}{L^{*}}$ obtained from Eq. 18 for different values of the coupling constant $\gamma$. The two scaling regimes at small and large $\frac{L}{L^{*}}$ are clearly visible.

In order to estimate the magnitude of the pseudo-Casimir forces in confined nematic polymers we now have to connect the macroscopic elastic constants $K_{1}, K_{3}, B$ and the correlation length $\xi$ with the microscopic parameters of the systems. This is in general a very difficult undertaking. For DNA this type of analysis has been performed in [11, 16] and we just quote these results.

If the interaction potential between the segments of the polymers is $U(D)$, where $D$ is the average spacing between the molecules perpendicular to the average director and the persistence length of the polymers is $\mathcal{L}_{P}$, one has [11]

$$
\begin{aligned}
K_{1}=K_{2} & \simeq U(D) / D \\
K_{3} & \simeq k T \mathcal{L}_{P} \rho_{\perp}+U(D) / D \\
B & \simeq \frac{\sqrt{3}}{4}\left(\frac{\partial^{2}}{\partial D^{2}}-\frac{1}{D} \frac{\partial}{\partial D}\right) U(D) / \mathcal{L}_{P} .
\end{aligned}
$$

One should realize here that since DNA is a polyelectrolyte the interaction potential $U(D)$ depends also on the ionic strength of the bathing solution.

This scaling of elastic constants leads to the conclusion that in Eqs. 21 and 22 we have $\frac{K_{2}}{K_{1}} \sim 1$ and $\frac{K_{3}}{K_{1}} \gg 1$. Thus the larger the $D$, i.e. the smaller the density of the polymers, the larger this ratio becomes. The magnitude of the pseudo-Casimir interaction thus depends fundamentally on the equation of state of the nematic polymers through the osmotic compressibility $B$ and the density perpendicular to the average nematic director, $\rho_{\perp}$.

Fig. 1 presents a plot of dimensionless free energy as a function of dimensionless separation. The curves are plotted for various values of the coupling constant $\gamma$ which is plotted as a function of the average spacing between polymers on Fig. 2 for the case of DNA. One can clearly 


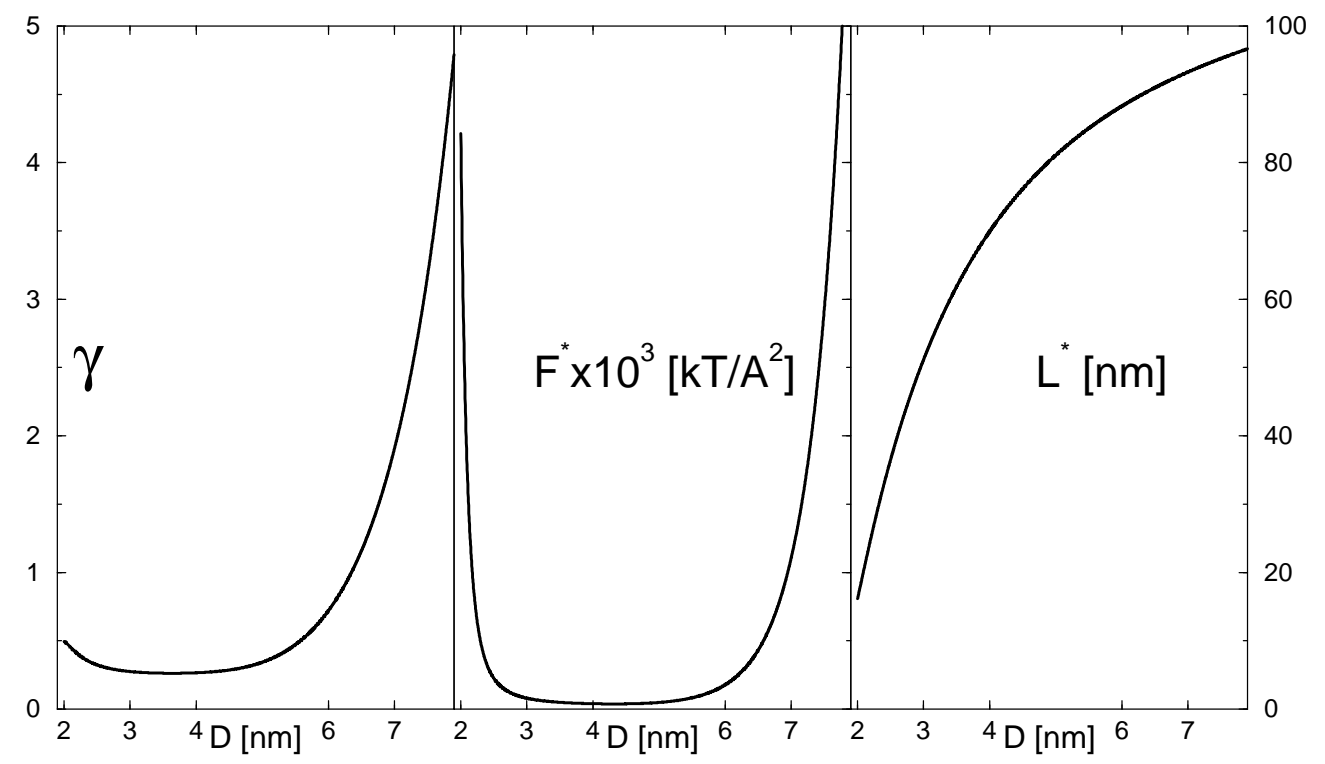

Fig. 2 - The dependence of the magnitude of the pseudo-Casimir interaction $\mathcal{F}^{*}$, the characteristic length $L^{*}$ and the coupling constant $\gamma$ on the average separation between the polymers obtained from [11] in the case of $0.5 \mathrm{M}$ bathing ionic solution. Note that $\gamma$ and $\mathcal{F}^{*}$ have the same scale. $L^{*}$ saturates for small values of DNA density (large $D$ ) at about $140 \AA$.

discern two regions with approximately $L^{-2}$ and $L^{-4}$ behavior separated by a characteristic spacing $L_{0}=C(\gamma) L^{*}$, where $C(\gamma)$ is a numerical factor depending on the coupling constant that can be read off Fig. 1. The numerical evidence shows that the caracteristic spacing $L_{0}$ grows approximately linearly with the average separation between the polymers $D$, ranging from about 15 to $20 \mathrm{~nm}$ at $D=2 \mathrm{~nm}$ to around $150 \mathrm{~nm}$ at $D=8 \mathrm{~nm}$, which is between one and two correlation lengths $\xi$.

Fig. 2 shows the dependence of the magnitude of the pseudo-Casimir interaction $\mathcal{F}^{*}$, the characteristic length $L^{*}$ and the coupling constant $\gamma$ on the average separation between the polymers obtained from the measured equation of state for DNA in the case of $0.5 \mathrm{M}$ bathing electrolyte solution (for details see [1] ).

One would of course like to have also approximate analytical formulae for the pseudoCasimir interaction Eq. 18. There are only two limiting cases where the integral in Eq. 18 can be evaluated analytically, the large separation and the small separation regime. First of all for small $L$ the dominant behavior of the integral is obtained at large $Q$ leading in this case to

$$
\mathcal{F}\left(L \ll L_{0}\right)=-\frac{k T S}{16 \pi L^{2}}\left(\frac{K_{3}}{K_{1}(1+4 \Lambda \xi) \cdot\left(1+\frac{B}{K_{1}} \xi^{2}\right)}+\frac{K_{2}}{K_{1}}\right) \zeta(3) .
$$

The other analytically tractable limit is in the case of large $L$ where the dominant contribution to the integral comes from small $Q$ behavior of the integrand

$$
\mathcal{F}\left(L \gg L_{0}\right)=-\frac{k T S}{16 \pi}\left[\frac{2 K_{3} \zeta(5)}{B L^{4}}+\frac{K_{2} \zeta(3)}{K_{1} L^{2}}\right],
$$


where the length scale $L_{0}$ can be read off the graph on Fig. 1 for various values of $\gamma$ (see above). Thus for small $L$ the confined polymer nematic behaves basically as a standard nematic [5] with a renormalized magnitude of the fluctuation interaction. For large $L$ the dependence is altogether different, deviating essentially from the standard nematic case. The crossover between the two regimes moves to larger $L$ as the polymers become less dense (see Fig. 2) but eventually saturates for small polymer densities.

The pseudo-Casimir interaction for long nematic polymers thus differs qualitatively from the one obtained in the case of short nematics [5]. Though it still decays algebraically with $L$, and thus qualifies as a long range force, it decays faster $\left(L^{-4}\right)$ then in the case of short nematics $\left(L^{-2}\right)$. Nevertheless its absolute magnitude, depending on the equation of state of the nematic polymer and the regime of spacings $L$, can become comparable to and even larger than in the case of short nematics with the same Frank constants. The $L^{-4}$ behavior stems essentially from the polymer stiffness and should thus be a salient feature of the pseudoCasimir interactions whenever the mesoscopic hamiltonian contains the squares of higher then the first derivatives in the order parameter. These type of systems will be studied in our future work.

We would like to thank P. Ziherl for his valuable comments on an earlier version of the MS.

\section{REFERENCES}

[1] V.M. Mostepanenko and N.N. Trunov, The Casimir Effect and its Applications (Clarendon Press, Oxford) 1997

[2] H. B. G. Casimir, Proc. Kon. Ned. Akad. Wet., 51 (1948) 793

[3] M. Krech, The Casimir effect in critical systems (World Scientific, Singapore) 1994

[4] H. Li and M. Kardar, Phys. Rev. Lett, 67 (1991) 3275 Phys. Rev. A, 46 (1992) 6490

[5] A. Ajdari, L. Peliti, and J. Prost, Phys. Rev. Lett, 66 (1991) 1481 A. Ajdari, B. DuPlantier, D. Hone, L. Peliti, and J. Prost, J. Phys. II France, 2 (1992) 487

[6] P. Ziherl, F. Karimi Pour Haddadan, R. Podgornik and S. Žumer, Phys. Rev., E , 61 (2000) 5361

[7] B. D. Swanson and L. B. Sorensen, Phys. Rev. Lett., 75 (1995) 3293

[8] I. MušEvič, G. Slak, and R. Blinc, Abstract Book of $16^{\text {th }}$ International Liquid Crystal Conference (Kent, Ohio) 1996, p. 91

[9] F. Vandenbrouck, M. P. Valignat, and A. M. Cazabat, Phys. Rev. Lett., 82 (1999) 2693

[10] P. Ziherl, R. Podgornik and S. Žumer, Phys. Rev. Lett., 84 (2000) 1228

[11] H.H. Strey, V.A. Parsegian and R. Podgornik, Phys. Rev. E, 59 (1999) 999

[12] P. G. De Gennes and J. Prost, The Physics of Liquid Crystals (Clarendon Press, Oxford) 1993

[13] P.G. De Gennes, Mol. Cryst. Liq. Cryst. Lett., 34 (177) 1977

[14] H. Kleinert, J. Math. Phys., 27 (3003) 1986

[15] C. Grosche and F.Steiner, Handbook of Feynman path integrals, Springer tracts in modern physics ; 145 (Springer, New York,) 1998

[16] H.H. Strey, J. Wang, R. Podgornik, A. Rupprecht, L. Yu, V.A. Parsegian, E. Sirota, Phys. Rev. Lett., 84 (2000) 3105 